\title{
An Adaptive Learning and Classifier Model in MRI Tumor Detection
}

\author{
Somashekhar Swamy \\ Research scholar \\ V.T.U.Belagavi, Karnataka, India.
}

\author{
P. K. Kulkarni \\ Professor \& H.O.D.(E\&EE), \\ P.D.A.College of Engineering. Kalburgi, \\ Karnataka, India.
}

\begin{abstract}
In the process of image coding, external noises impact a lot in processing efficiency. In the application of medical image processing, this effect is more, important due to its finer content details. It is required to minimize the noise effect with preserving the image content information, without losing the image generality. Towards the objective of image denoising, in this work, a dynamic block coding approach for noise minimization in medical image processing is presented. The filtration approach is an enhancement to the objective of noise elimination using median filtration. The suggested approach, improves the retrieval accuracy more effectively under variant noise condition in consideration to conventional filtration approach.
\end{abstract}

\section{General Terms}

Pattern recognition, medical image processing, tumor detection

\section{Keywords}

Denoising, medical image processing, dynamic block coding, MRI images.

\section{INTRODUCTION}

Current developments have led to attaining higher coding efficiency in image processing applications and its utilization. In different level of applications, medical image processing has its own importance. In the area of medial image coding, finer details coding and preservation is of prime importance. Towards the accuracy in image coding, various approaches were developed in past, to achieve the objective of image quality improvement. Various well-established techniques, such as median filtering are successfully used in gray scale imaging. Median filtering approach is particularly adapted for impulsive noise suppression. It has been shown that median filters present the advantage to remove noise without blurring edges since they are nonlinear operators of the class of rank filters and since their output is one of the original gray values [1][2]. The extension of the concept of median filtering to color images is not trivial. The main difficulty in defining a rank filter in color image is that there is no "natural" and unambiguous order in the data [3][4]. During the last years, different methods were proposed to use median filters in color medical image processing [5][6]. Whatever the vector filtering method, the challenge is to detect and replace noisy pixels whereas the relevant information is preserved. But it is recognized that in some MRI image areas most of vector filters blur thin details and image edges [7][8][9]. Generally impulse noise contaminates medical images during data acquisition by camera sensors and transmission in the communication channel. [10] proposed a two-phase algorithm. In the first phase of this algorithm, an adaptive median filter (AMF) is used to classify corrupted and uncorrupted pixels; in the second phase, specialized regularization method is applied to the noisy pixels to preserve the edges and noise suppression. The main drawback of this method is that the processing time is very high because it uses a very large window size of $39 \times 39$ in both phases to obtain the optimum output; in addition, more Complex circuitry is needed for their implementation. [11] proposed a sorting based algorithm in which the corrupted pixels are replaced by either the median pixel or neighborhood pixel in contrast to AMF and other existing algorithms that use only median values for replacement of corrupted pixels. At higher noise densities this algorithm does not preserve edge and fine details satisfactorily. In this paper a novel robust estimation based filter is proposed to remove fixed value impulse noise effectively. The proposed filter removes low to high density fixed value impulse noise with edge and detail preservation upto a noise density of $90 \%$. Recently, nonlinear estimation techniques are gaining popularity for the problem of image denoising. The well-known Wiener filter for minimum meansquare error (MMSE) estimation is designed under the assumption of wide-sense stationary signal and noise a random process is said to be stationary when its statistical characteristics are time domain invariant [12]. For most of the natural MRI images, the stationary condition is not satisfied. In the past, many of the noise removing filters were designed with the stationary assumption. These filters remove noise but tend to blur edges and fine details. This algorithm fails to remove impulse noise in high frequency regions such as edges in the MRI image. To overcome the above mentioned difficulties a nonlinear estimation technique for the problem of medical image denoising has been developed based on robust statistics. Robust statistics addresses the problem of estimation when the idealized assumptions about a system are occasionally violated. The contaminating noise in an image is considered as a violation of the assumption of time domain coherence of the medical image intensities and is treated as an outlier random variable [12]. [13] Developed a robust parameter estimation algorithm for the medical image model that contains a mixture of Gaussian and impulsive noise. In [12] a robust estimation based filter is proposed to remove low to medium density Gaussian noise with detail preservation. Though these techniques were developed for filtration of Gaussian or impulsive noise they are been developed for gray level images and are not suitable for color images. In this paper a modified approach to time domain median filter is proposed for the noise removal in digital medical images. The paper is further presented in six sections. Where conventional time domain filtration methods and their limitations were presented in Section 2. Section 3 outlines the proposed modified median filtration approach for MRI images. The simulation observations were presented in section 4.

\section{PROPOSED SYSTEM OUTLINE}

In the approach of medical image processing, automated image recognition for tumor detection has its own significance. And automate system can provide a early stage 
analysis and decision based on the image data passed with more effective way. An approach of automated processing medical image data analysis system is presented in figure 1 . The system basically consists of a preprocessing stage, feature extraction and classification stage. The primary requirement of any image coding system is to process the image to an extent of maximum accuracy retaining the image integrity.

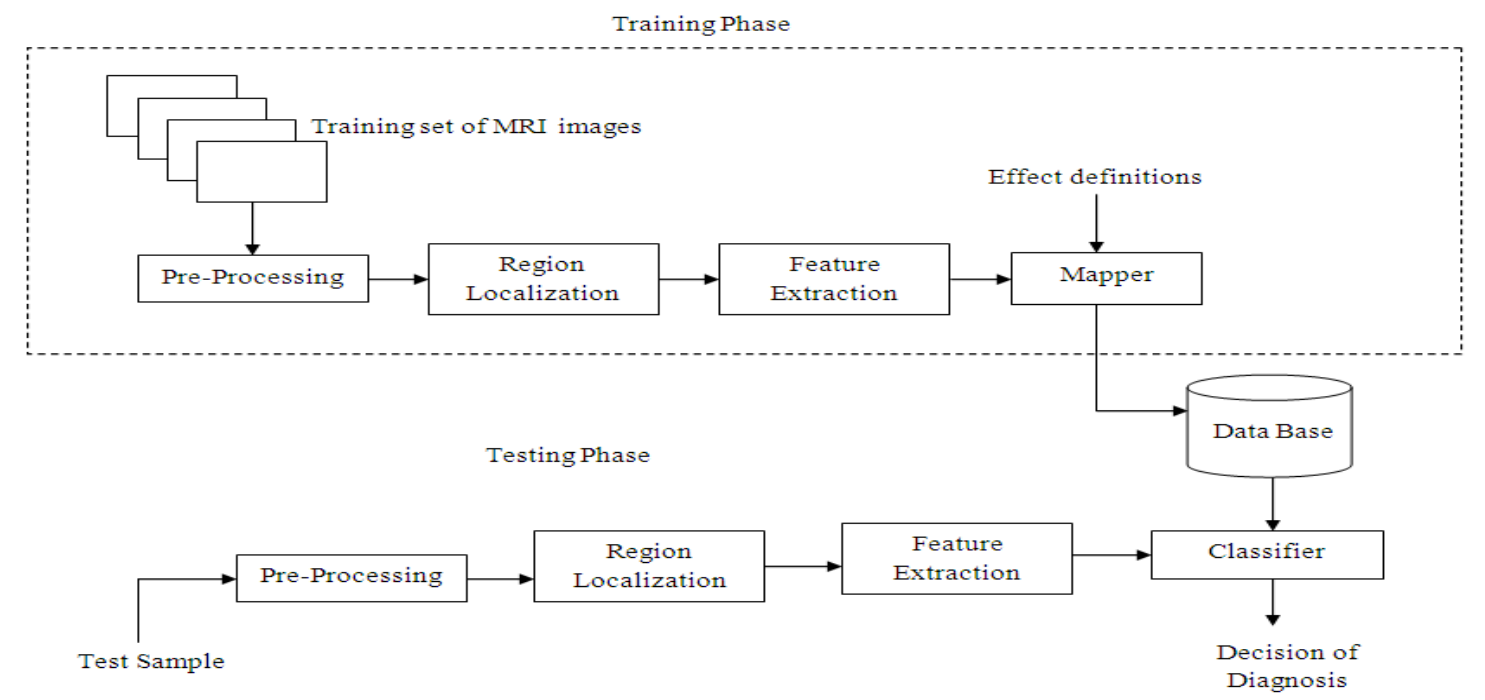

Figure 1: Proposing system architecture for the automated Diagnosis system

In the pre-processing unit the given sample is processed for a standard processing size, extracting the pixel values and performing filtration to eliminate noise effects. The process of denoising was observed in various literatures to eliminate noise effects at preprocessing level. In recent approach towards denoising of MRI sample at preprocessing median filtration was suggested [3]. Wherein median filtration are effective under a discrete level of noise effect, under dynamic noise variations the immunity is reduced. In the operation of median filtration, The values of the pixel in the window are stored and the median - the middle value in the sorted list (or average of the middle two if the list has an even number of elements)-is the one plotted into the output image. The median filtered image $\mathrm{g}(\mathrm{x}, \mathrm{y})$ can be obtained from the median pixel values in a neighborhood of $(x, y)$ in the input image $f(x, y)$, as defined by the following formula:

$$
\begin{gathered}
\operatorname{MdF}\left(x_{i}\right)=\operatorname{Median}\left(\left\|x_{i}\right\|^{2}\right) \\
\text { Where, } \mathrm{i}=1 \ldots \mathrm{N}
\end{gathered}
$$

These filtration techniques were found to be effective in gray scale images. When processed over color images these filtration techniques give lesser performance. To achieve accurate reconstruction of medical image the median filtration technique is modified to time domain median filtration. The Time domain Median Filter is a uniform smoothing algorithm with the purpose of removing noise and fine points of medical image data while maintaining edges around larger shapes.

\section{ADAPTIVE LEARNING APPROACH}

In a Time domain Median Filter the vectors are ranked by some criteria and the top ranking point is used to the replace the center point. No consideration is made to determine if that center point is original data or not. The unfortunate drawback to using these filters is the smoothing that occurs uniformly across the image. Across areas where there is no noise, original medical image data is removed unnecessarily. In the Modified Time domain Median Filter, after the time domain depths between each point within the mask are computed, an attempt is made to use this information to first decide if the mask's center point is an uncorrupted point. If the determination is made that a point is not corrupted, then the point will not be changed.

The proposed modified filtration works as explained below,

1) Calculate the time domain depth of every point within the mask selected.

2) Sort these time domain depths in descending order.

3) The point with the largest time domain depth represents the Time domain Median of the set. In cases where noise is determined to exist, this representative point is used to replace the point currently located under the center of the mask.

4) The point with the smallest time domain depth will be considered the least similar point of the set.

5) By ranking these time domain depths in the set in descending order, a time domain order statistic of depth levels is created.

6) The largest depth measures, which represent the collection of uncorrupted points, are pushed to the front of the ordered set.

7) The smallest depth measures, representing points with the largest time domain difference among others in the mask and possibly the most corrupted points, and they are pushed to the end of the list. This prevents the smoothing by looking for the position of the center point in the time domain order statistic list. The image inter relation error is then minimize using a least mean error (LMSE) estimation. The Least Mean Square (LMS) algorithm is an adaptive algorithm, which uses a gradient-based method of steepest decent. LMS algorithm uses the estimates of the gradient vector from the available data. LMS incorporates an iterative procedure that makes successive corrections to the weight vector in the direction of the negative of the gradient vector, which eventually leads to the minimum mean square error. Compared to other algorithms LMS algorithm is relatively 
simple; it does not require correlation function calculation nor does it require matrix inversions. From the method of steepest descent, the weight vector equation is given by;

$$
w(n+1)=w(n)+1 / 2 \mu\left[-\Delta\left(E\left\{e^{2}(n)\right\}\right)\right]
$$

Where $\mu$ is the step-size parameter and controls the convergence characteristics of the LMS algorithm; $\mathrm{e}^{2}(n)$ is the mean square error between the output $y(n)$ and the reference signal which is given by,

$$
e^{2}(n)=\left[d^{*}(n)-w^{h} x(n)\right]^{2}
$$

The gradient vector in the above weight update equation can be computed as

$$
\Delta_{w}\left(E\left\{e^{2}(n)\right\}\right)=-2 r+2 R w(n)
$$

In the method of steepest descent the biggest problem is the computation involved in finding the values $r$ and $R$ matrices in real time. The LMS algorithm on the other hand simplifies this by using the instantaneous values of covariance matrices $r$ and $\mathrm{R}$ instead of their actual values i.e.

$$
\begin{aligned}
& R(n)=x(n) x^{h}(n) \\
& R(n)=x(n) d^{*}(n)
\end{aligned}
$$

Therefore the weight update can be given by the following equation,

$$
W(n+1)=w(n)+\mu x(n)\left[d^{*}(n)-w(n) x^{h}(n)\right](7)
$$

The LMS algorithm is initiated with an arbitrary value $\mathrm{w}(0)$ for the weight vector at $n=0$. The successive corrections of the weight vector eventually leads to the minimum value of the mean squared error. Therefore the LMS algorithm can be summarized in following equations;

$$
\begin{aligned}
& \text { Output, } \left.(n)=x(n) w^{h}(n)\right] \\
& \text { Error, } e(n)=d^{*}(n)-y(n) \\
& \text { Weight, } w(n+1)=w(n)+\mu x(n) e^{*}(n)
\end{aligned}
$$

This computed weight provides an optimal value for noise elimination. Using this noise limit, the images are Denoised and passed for higher grid interpolation. The experimental result obtained for the developed system is as illustrated in the following section.

\section{SVM CLASSIFIER MODEL}

In machine learning, support vector machines (SVMs, also support vector networks) are supervised learning models with associated learning algorithms that analyze data and recognize patterns, used for classification and regression analysis. Given a set of training examples, each marked for belonging to one of two categories, an SVM training algorithm builds a model that assigns new examples into one category or the other, making it a nonprobabilistic binary linear classifier. An SVM model is a representation of the examples as points in space, mapped so that the examples of the separate categories are divided by a clear gap that is as wide as possible. New examples are then mapped into that same space and predicted to belong to a category based on which side of the gap they fall on. In addition to performing linear classification, SVMs can efficiently perform a non-linear classification using what is called the kernel trick, implicitly mapping their inputs into high-dimensional feature spaces.More formally, a support vector machine constructs a hyper plane or set of hyper planes in a high- or infinite-dimensional space, which can be used for classification, regression, or other tasks. Intuitively, a good separation is achieved by the hyper plane that has the largest distance to the nearest training-data point of any class (socalled functional margin), since in general the larger the margin the lower the generalization error of the classifier.Whereas the original problem may be stated in a finite dimensional space, it often happens that the sets to discriminate are not linearly separable in that space. For this reason, it was proposed that the original finite-dimensional space be mapped into a much higher-dimensional space, presumably making the separation easier in that space. To keep the computational load reasonable, the mappings used by SVM schemes are designed to ensure that dot products may be computed easily in terms of the variables in the original space, by defining them in terms of a kernel function $\mathrm{k}(\mathrm{x}, \mathrm{y})$ selected to suit the problem. The hyper planes in the higherdimensional space are defined as the set of points whose dot product with a vector in that space is constant. The vectors defining the hyper planes can be chosen to be linear combinations with parameters $\alpha_{i}$ of images of feature vectors $x_{i}$ that occur in the data base. With this choice of a hyper plane, the points $X$ in the feature space that are mapped into the hyper plane are defined by the relation:

$$
\sum_{i} \alpha_{i} k\left(x_{i}, x\right)=\text { constant }
$$

Note that if $\mathrm{k}(\mathrm{x}, \mathrm{y})$ becomes small as $\mathrm{y}$ grows further away from $x$, each term in the sum measures the degree of closeness of the test point $\chi$ to the corresponding data base point $x_{i}$. In this way, the sum of kernels above can be used to measure the relative nearness of each test point to the data points originating in one or the other of the sets to be discriminated. Note the fact that the set of points $\chi$ mapped into any hyper plane can be quite convoluted as a result, allowing much more complex discrimination between sets which are not convex at all in the original space.

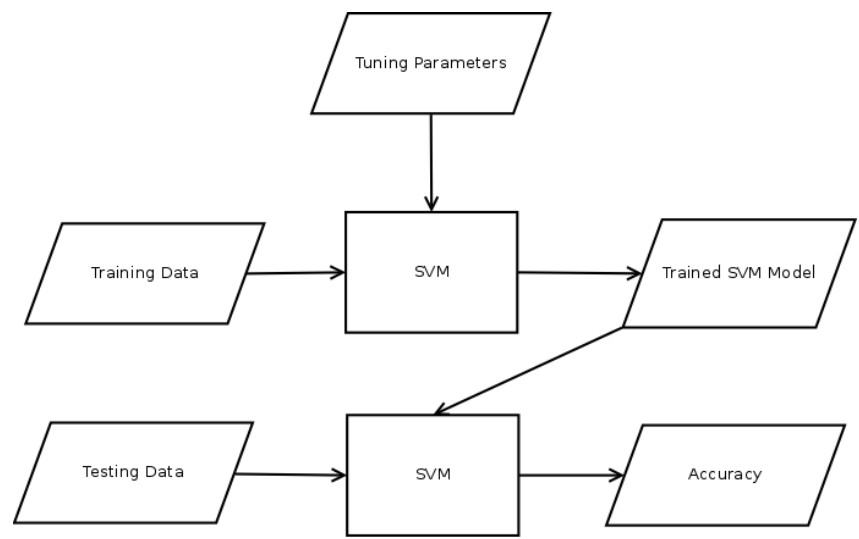

Fig.2: SVM classifier working

\section{EXPERIMENTAL RESULTS}

To test the accuracy of the modified time domain median filter, a medical image with corruption applied by some means is applied. To estimate the quality of a reconstructed MRI image, first calculate the Root-Mean-Squared Error between the original and the reconstructed image. The Root-MeanSquared Error (RMSE) for an original image $\mathrm{I}$ and reconstructed MRI image $\mathrm{R}$ is defined by,

$$
\operatorname{RMSE}(I, R)=\sqrt{\frac{1}{I_{w} \times I_{h}}} \sum_{i=0}^{I_{W}} \sum_{j=0}^{I_{h}}\|(i, j)-R(i, j)\|^{2}
$$


The algorithm for the Modified Time domain Median Filter (MSMF) requires two parameters. The first parameter considered is the size of the mask to use for each filtering operation. The second parameter, threshold $\zeta$, represents the estimated number of original points for any given sample under a mask. A collection of ten MRI images of various sizes was used in these tests. These images are a variety of textures and subject matter. The texture of these MRI images impact on the threshold chosen than the window mask size.

The tests to determine the best mask size were conducted in this manner:

1. Each of the ten MRI images in the collection was artificially distorted with $\rho=0.0, \rho=0.05, \quad \rho$ $=0.10$, and $\rho=0.20$ noise composition, resulting in 40 images.

2. Each of the forty medical noisy images was then reconstructed using the SMF with mask sizes of $\mathrm{N}=3, \mathrm{~N}=5$, and $\mathrm{N}=7$ (the second argument, threshold $\zeta$, is set to 1 ), resulting in 120 reconstructed medical images.

3. The Root-Mean-Squared Error was computed between all 120 reconstructed MRI images and the originals. The RMSE is a simple estimation score of the difference between two MRI images. An ideal RMSE would be zero, which means that the algorithm correctly identified each noisy point and also correctly derived the original data at that location in the signal. For the evaluation of the work a performance evaluation is carried out for various samples and the result obtained were as shown below

For the evaluation of the work a performance evaluation is carried out for various samples and the results obtained were as shown below. As seen in figure 2, a mask size of 3 clearly outperformed the other tested sizes of 5 and 7. Neither the amount of noise, the size of the MRI image, nor the subject matter of the image effects on which mask size performed the best. Less thorough tests were run on higher mask sizes such as 9 and 11. With each increase in mask size, the RMSE of each test increased. Most of the medical images are images of various scenes, such as portrait shots, nature shots, animal shots, scenic shots of snow-capped mountains and sandy beaches. When comparing the Adaptive Masking Filter, the Adaptive Masking Filter, the Masking Filter, and the Mean Filter, a 2000 image subset of the 59,895 images were used.

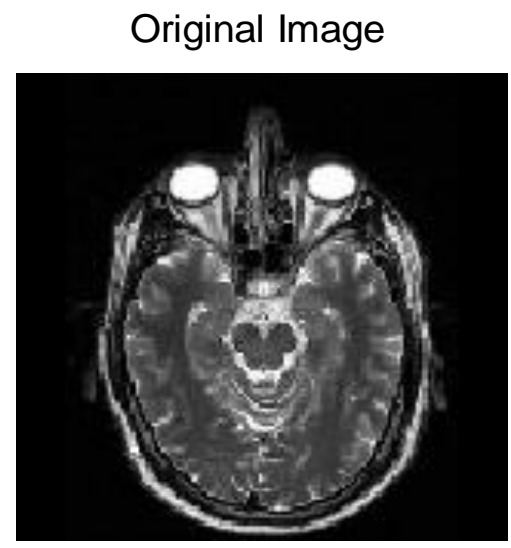

(a)

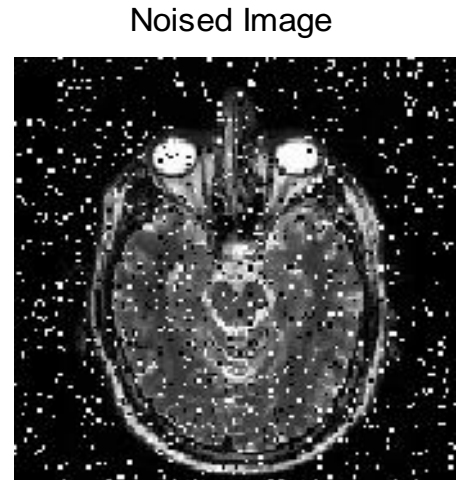

(b)

Mean filtered

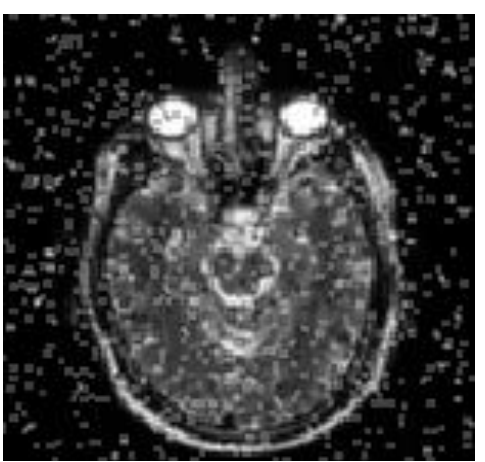

(c)

Median Filtered

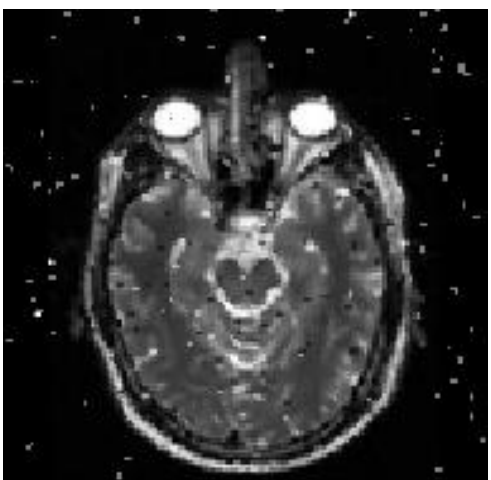

(d)

\section{Adaptive Mask filtered}

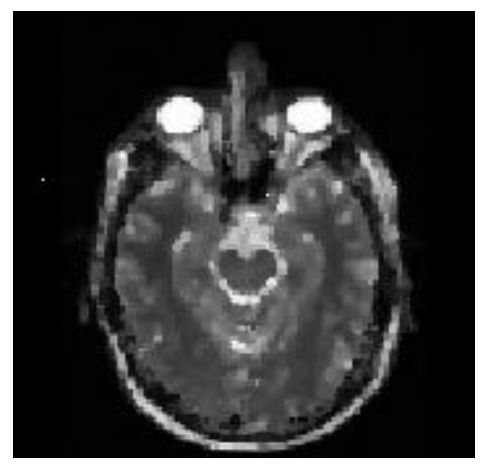

(e)

Fig 3 (a) Original MRI sample for processing (b) noised image sample at variance of 0.1 (c) mean filter output of noise image sample (d) median filter output for the same noised sample (e) obtained filtered output using proposed AMF filtration 
Fig 3 illustrates the obtained result observation for given MRI sample, affected by salt pepper noise at a variance of 0.1 . The estimation using Adaptive mask filter is observed to be more effective in estimation in comparison to the conventional filtration approaches. Due to the usage of block mask processing, the surrounding pixels were processed with low region noise distribution in comparison to the existing filtration approach.

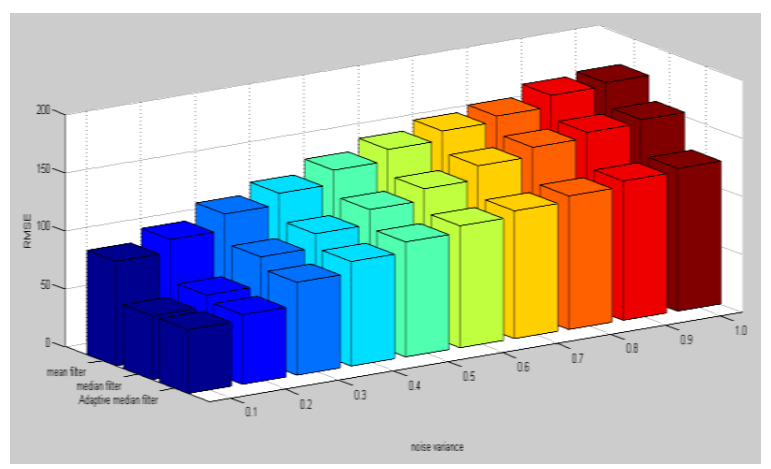

Fig 4 comparative variation of obtained root mean square value over noise variation for the masking length of 3 for the three filters

The Obtained RMSE estimation approach shows that with the increase in noise variance, the obtained root mean square error for the proposed AMF filtration is comparatively lower than the other two conventional approaches. With the increase in the noise variance to the input signal, it is observed that RMSE effectively falls down almost to $1 / 2$ for median filter and double for mean filter.

Table 1: Observation for obtained RMS value over different noise variance for the given sample

\begin{tabular}{|c|c|c|c|}
\hline $\begin{array}{c}\text { Noise } \\
\text { variance }\end{array}$ & $\begin{array}{c}\text { RMSE(mean } \\
\text { filter) }\end{array}$ & $\begin{array}{c}\text { RMSE(median } \\
\text { filter) }\end{array}$ & $\begin{array}{c}\text { RMSE } \\
\text { (adaptive ) }\end{array}$ \\
\hline 0.1 & 90 & 55 & 55 \\
\hline 0.2 & 101 & 65 & 60 \\
\hline 0.3 & 115 & 90 & 80 \\
\hline 0.4 & 125 & 102 & 90 \\
\hline 0.5 & 137 & 115 & 99 \\
\hline 0.6 & 147 & 125 & 105 \\
\hline 0.7 & 155 & 137 & 110 \\
\hline 0.8 & 160 & 145 & 115 \\
\hline 0.9 & 170 & 150 & 120 \\
\hline 1.0 & 173 & 153 & 123 \\
\hline
\end{tabular}

AM-Filtered at $\mathrm{N}=2$

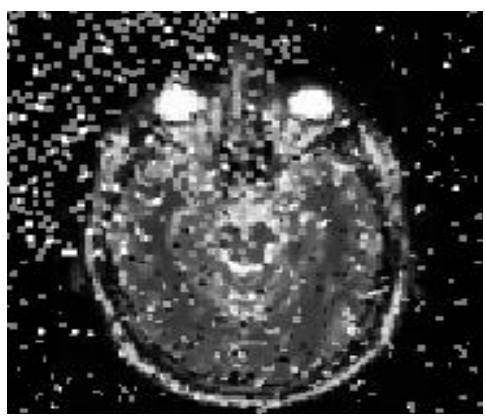

(a)

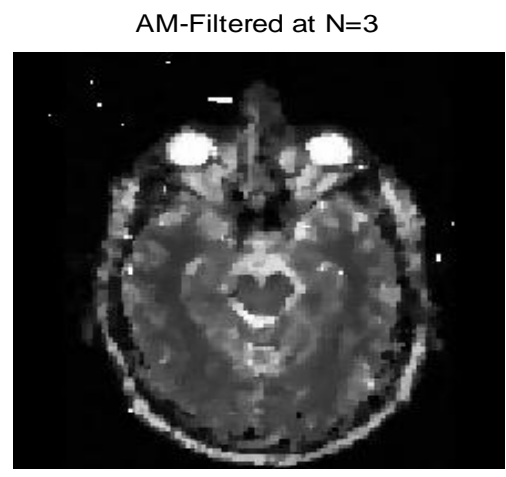

(b)

AM-Filtered at $\mathrm{N}=4$

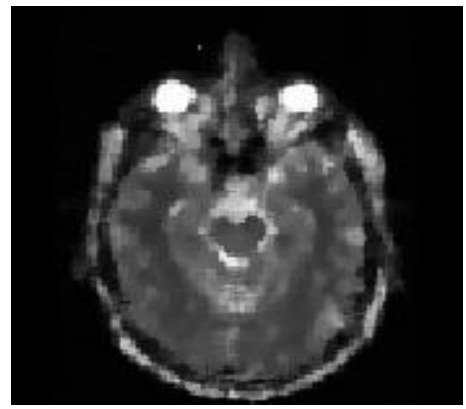

(c)

Fig 5 (a) filtered output at $\mathrm{N}=2$ for the proposed $\mathrm{AMF}$ filter (b) Result at $\mathrm{N}=3$ (c) Result at $\mathrm{N}=4$

The figure illustrates the obtained result observations with the variation in block size $(\mathrm{N})$ for the Adaptive mask filters. The result at $\mathrm{N}=3,4$ is observed to be more accurate than and $\mathrm{N}=2$ and get saturated at $\mathrm{N}=5$.

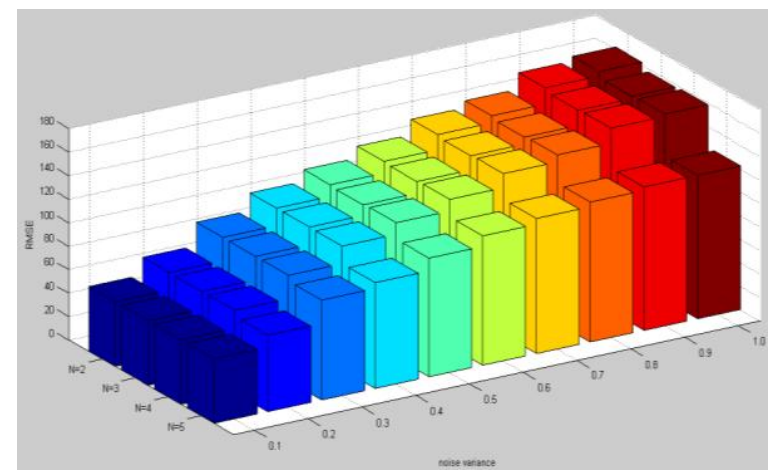

Fig.6 RMS observed for the AMF filter at different block lengths for Noise variance of 0.1 to 1 .

The observation made for the RRMSE value at different noise variance with the change in block size $(\mathrm{N})$ is presented in figure 4.4. The RMSE value for the test MRI sample is observed comparatively very low at $\mathrm{N}=5$, for high noise variance in the image.

Table 2 Observation of RMS for different noise variance

\begin{tabular}{|c|c|c|c|c|}
\hline $\begin{array}{c}\text { Noise } \\
\text { variance }\end{array}$ & RMSE(N=2) & RMSE(N=3) & RMSE(N=4) & $\begin{array}{c}\text { RMSE } \\
(\mathbf{N = 5})\end{array}$ \\
\hline 0.1 & 55 & 55 & 55 & 55 \\
\hline 0.2 & 70 & 70 & 70 & 65 \\
\hline 0.3 & 90 & 90 & 90 & 85 \\
\hline 0.4 & 105 & 105 & 105 & 90 \\
\hline 0.5 & 115 & 114 & 115 & 101 \\
\hline
\end{tabular}




\begin{tabular}{|l|l|l|l|l|}
\hline 0.6 & 125 & 124 & 125 & 110 \\
\hline 0.7 & 138 & 136 & 137 & 115 \\
\hline 0.8 & 144 & 142 & 143 & 119 \\
\hline 0.9 & 158 & 155 & 156 & 122 \\
\hline 1.0 & 162 & 157 & 158 & 122 \\
\hline
\end{tabular}

To evaluate the performance of the developed approach following parameters are used.

$$
\text { Accuracy }=\frac{T P+T N}{T P+T N+F P+F N}
$$

Where,

$$
\begin{aligned}
& \mathrm{TP}=\text { True positive (Correctly identified) } \\
& \mathrm{FP}=\text { False positive (Incorrectly identified) } \\
& \mathrm{TN}=\text { True negative (false, } \text { Correctly identified) } \\
& \mathrm{FN}=\text { False negative (false, incorrectly identified) }
\end{aligned}
$$

For the given simulation model, four classes with each class having 5 subjects, forming total of 20 subjects is used for training. During testing process, a query sample is given and the extracted features are passed to SVM classifier.

Along with accuracy, to show the enhancement of propose approach and also to compare the proposed approach with earlier approaches, few more metrics such as sensitivity, specificity, Recall, precision and F-measure was evaluated with following mathematic expressions.

Sensitivity measures the proportion of positives that are correctly identified as such.

$$
\text { Sensitivity }=\frac{T P}{T P+F N}
$$

Specificity measures the proportion of negatives that are correctly identified as such.

$$
\text { Specificity }=\frac{T N}{T N+F P}
$$

Precision is the fraction of identified instances that are correct, while recall is the fraction of correct instances that are identified.

$$
\text { Recall }=\frac{T P}{T P+F N}
$$

Table 4.Parametric evaluation of the developed system for processing efficiency.

\begin{tabular}{|c|c|c|c|c|c|c|c|c|}
\hline $\begin{array}{c}\text { Test } \\
\text { sample }\end{array}$ & DR-method & $\begin{array}{c}\text { Accuracy } \\
(\%)\end{array}$ & Sensitivity & Specificity & Recall & Precision & F-Measure & CT \\
\hline \multirow[b]{3}{*}{ Class 1} & Mean & 55.670 & 0.220 & 0.608 & 0.220 & 0.680 & 0.478 & 0.545 \\
\hline & Median & 62.500 & 0.315 & 0.752 & 0.315 & 0.740 & 0.523 & 0.348 \\
\hline & Adaptive & 70.000 & 0.444 & 0.909 & 0.444 & 0.800 & 0.571 & 0.138 \\
\hline \multirow[b]{3}{*}{ Class 2} & Mean & 49.484 & 0.432 & 0.712 & 0.432 & 0.508 & 0.542 & 0.273 \\
\hline & Median & 58.1341 & 0.458 & 0.854 & 0.458 & 0.666 & 0.621 & 0.143 \\
\hline & Adaptive & 69.500 & 0.524 & 0.946 & 0.524 & 0.820 & 0.652 & 0.137 \\
\hline \multirow[b]{3}{*}{ Class 3} & Mean & 55.670 & 0.420 & 0.762 & 0.420 & 0.650 & 0.569 & 0.310 \\
\hline & Median & 63.824 & 0.452 & 0.886 & 0.452 & 0.720 & 0.688 & 0.139 \\
\hline & Adaptive & 70.840 & 0.484 & 0.924 & 0.484 & 0.795 & 0.690 & 0.132 \\
\hline \multirow[b]{3}{*}{ Class 4} & Mean & 58.360 & 0.446 & 0.738 & 0.446 & 0.650 & 0.583 & 0.374 \\
\hline & Median & 65.420 & 0.558 & 0.824 & 0.558 & 0.745 & 0.600 & 0.183 \\
\hline & Adaptive & 72.820 & 0.582 & 0.908 & 0.582 & 0.810 & 0.680 & 0.132 \\
\hline
\end{tabular}

$$
\text { Precision }=\frac{T P}{T P+F P}
$$

F-measure or balanced F-score is a measure that combines precision and recall is the harmonic mean of precision and recall.

$$
F_{\_} \text {measure }=\frac{2 * \text { Recall.Precision }}{\text { Recall }+ \text { Precision }}
$$

The obtained retrieval observations for different test action in the Weizmann dataset were observed through the feature count and the overhead.

\section{CONCLUSION}

In this paper an adaptive learning system for automated detection and classification of tumor in brain image is presented. A common noise filtering algorithms were discussed. Next, a Spatial Median Filter was proposed based on a combination of work on the Median Filter and the Spatial Median quantile order statistic. Seeing that the order statistic could be utilized in order to make a judgment as to whether a point in the signal is considered noise or not, a Modified Spatial Median Statistic is proposed. The Modified Median Filter operates on two parameters: A window size and a threshold $\mathrm{T}$ of the estimated non-noisy pixels under a mask. In the results, the best threshold $\mathrm{T}$ to use in the Modified Spatial Median Filter and determined that the best threshold is 4 when using a $3 \times 3$ window mask size. The noise free samples are more accurate in tumor region localization and detection based on the spectral feature and SVM classifier model.

\section{REFERENCES}

[1] M. H. C. Lakshmi Devasena, "Noise Removal in Magnetic Resonance Images using Hybrid KSL Filtering Technique," Int. J. Comput. Appl., vol. 27, no. 8, pp. 1-4, 2011.

[2] M. A. Yousuf and M. N. Nobi, "A New Method to Remove Noise in Magnetic Resonance and Ultrasound Images," J. Sci. Res., vol. 3, no. 1, pp. 81-88, Dec. 2010. 
[3] D. Ray, D. Dutta Majumder, and A. Das, "Noise reduction and image enhancement of MRI using adaptive multiscale data condensation," 2012 1st Int.Conf. Recent Adv. Inf. Technol., pp. 107-113, Mar. 2012.

[4] M. R. Jose V. Manjon, Pierrick Coupe, AntoniBuades, D Louis Collins, "New methods for MRI denoising based on sparseness and self-similarity," Med. Image Anal., vol. 16, pp. 18-27, 2012.

[5] J. M. Waghmare and B. D. Patil, "Removal of Noises In Medical Images By Improved Median Filter," Int. J. Eng. Sci., vol. 2, no. 7, pp. 49-53, 2013.

[6] T. Rajeesh, J., Moni, R. S., Palanikumar, S., \&Gopalakrishnan, "Noise Reduction in Magnetic Resonance Images using Wave Atom Shrinkage," Int. J.Image Process., vol. 4, no. 2, pp. 131-141, 2010.

[7] M. R. Jose V. Manjon , Jose Carbonell-Caballero, Juan J. Lull, Gracian Garcia-Mart1, Lu1s Mart1-Bonmat1, "MRI denoising using Non-Local Means," Med. Image Anal., vol. 12, pp. 514-523, 2008.
[8] R. G. Hong Liua, Cihui Yang, Ning Pan, Enmin Song, "Denoising 3D MR images by the enhanced non-local means filter for Rician noise," Magn. Reson. Imaging, vol. 28, pp. 1485-1496, 2010.

[9] B. Shinde, D. Mhaske, M. Patare, a R. D. International, and a R. Dani, "Apply Different Filtering Techniques To Remove the Speckle Noise Using Medical Images," Int. J. Eng. Res. Appl., vol. 2, no. 1, pp. 1071-1079, 2012.

[10] M. K. S. Sivasundari, R. Siva Kumar, "Performance Analysis of Image Filtering Algorithms for MRI Images," Int. J. Res. Eng. Technol., vol. 3, no. 5, pp. 438-440, 2014.

[11] E. R. McVeigh, R. M. Henkelman, and M. J. Bronskill, "Noise and filtration in magnetic resonance imaging.," Med. Phys., vol. 12, no. 5, pp. 586-91, 1985.

[12] H. Gudbjartsson and S. Patz, "The Rician distribution of noisy MRI data.," Magn. Reson. Med., vol. 34, no. 6, pp. 910-4, Dec. 1995.

[13] R. E. W. R. C. Gonzalez, Digital Image Processing, Third Edit. Prentice Hall, 2007. 